PROCEEDINGS OF THE

AMERICAN MATHEMATICAL SOCIETY

Volume 128, Number 3, Pages 745-754

S 0002-9939(99)05033-9

Article electronically published on July 27, 1999

\title{
EXTENSIONS OF HOLOMORPHIC MAPS THROUGH HYPERSURFACES AND RELATIONS TO THE HARTOGS EXTENSIONS IN INFINITE DIMENSION
}

\author{
DO DUC THAI AND NGUYEN THAI SON
}

(Communicated by Steven R. Bell)

\begin{abstract}
A generalization of Kwack's theorem to the infinite dimensional case is obtained. We consider a holomorphic map $f$ from $Z \backslash H$ into $Y$, where $H$ is a hypersurface in a complex Banach manifold $Z$ and $Y$ is a hyperbolic Banach space. Under various assumptions on $Z, H$ and $Y$ we show that $f$ can be extended to a holomorphic map from $Z$ into $Y$. Moreover, it is proved that an increasing union of pseudoconvex domains containing no complex lines has the Hartogs extension property.
\end{abstract}

\section{INTRODUCTION}

We discuss the problems of extending holomorphic maps through hypersurfaces and extending to the envelope of holomorphy, i.e. the Hartogs holomorphic extension. These two problems are related.

In [10], Kwack showed that if $f$ is a holomorphic map from the punctured unit disc $\Delta^{*}$ into a hyperbolic manifold $X$ such that, for a suitable sequence of points $z_{k} \in \Delta^{*}$ converging to the origin, $f\left(z_{k}\right)$ converges to a point $p_{0} \in X$, then $f$ extends to a holomorphic map from the unit disc $\Delta$ into $X$.

The above-mentioned theorem of Kwack plays an essential role and has strongly motivated the study of the extension problem of holomorphic maps. Thus this was generalized in many directions. In particular, it also generalized to the problem of extending holomorphic maps through hypersurfaces; see, for instance, the survey [11].

Therefore proving Kwack type theorems in the infinite dimensional case is really necessary and motivates the study of the extension problem of holomorphic maps in infinite dimension.

In Sect. 1 we discuss the problem of determining when a holomorphic map can be extended through hypersurfaces in complex Banach manifolds. We prove the following theorems.

Theorem 1.1. Let $X$ be a hyperbolic Banach analytic space and $f: Z \backslash H \rightarrow X a$ holomorphic map, where $H$ is a hypersurface in a complex Banach manifold $Z$. Assume that for every $z \in H$ there exists a sequence $\left\{z_{n}\right\} \in Z \backslash H$ converging to $z$ such

Received by the editors May 27, 1997 and, in revised form, April 20, 1998.

1991 Mathematics Subject Classification. Primary 32E05, 32H20; Secondary 32F05, 58B12.

Supported by the State Program for Fundamental Research in Natural Science.

(C)1999 American Mathematical Society 
that the sequence $\left\{f\left(z_{k}\right)\right\}$ converges to $x_{z} \in X$. Then $f$ extends holomorphically to $Z$.

Theorem 1.2. Let $X$ be a hyperbolic Banach analytic space which is complete in the Cauchy mean and $f: Z \backslash H \rightarrow X$ a holomorphic map, where $H$ is a hypersurface in a complex Banach manifold $Z$. Assume that for every branch $H_{\alpha}$ of $H$ there exist $z_{\alpha} \in \operatorname{Reg}\left(H_{\alpha}\right)$ and a sequence $\left\{z_{n}^{\alpha}\right\}_{n=1}^{\infty} \subset Z \backslash H$ converging to $z_{\alpha}$ such that the sequence $\left\{f\left(z_{n}^{\alpha}\right)\right\}$ converges to $p_{\alpha} \in X$. Then $f$ extends holomorphically to $Z$.

We would like to emphasize here that the local compactness of (finite dimensional) complex manifolds plays an essential role in proving the finite dimensional Kwack theorems. Since complex Banach manifolds do not have the local compactness property, the technique for proving Kwack type theorems in the infinite dimensional case required substantial changes. The proofs of the above-mentioned theorems are based on the maximum principle for plurisubharmonic functions.

In Sect. 2 we go further. We would like to investigate deep interactions between the Brody hyperbolicity of Banach analytic spaces and the Hartogs extension property.

First we recall the definition of a (finite-dimensional) complex space having the Hartogs extension property (HEP for short) which was introduced by Ivashkovicz [7]. A complex space $X$ is said to have HEP if every holomorphic map $f$ from $\Omega$ into $X$, where $\Omega$ is a Riemann domain over $\mathbb{C}^{n}$, can be extended holomorphically to the envelope of holomorphy $\widehat{\Omega}$ of $\Omega$. This definition can be generalized naturally to the case of Banach analytic spaces by replacing $\mathbb{C}^{n}$ by an arbitrary Banach space $B$. However, for technical reasons we need the statement that every pseudoconvex Riemann domain over a Banach space $B$ is a domain of existence. One only was proved in the case that the Banach space $B$ has a Schauder basis (see Mujica [13, Theorem 54.12, p. 390]). Thus in the infinite-dimension case we give the following definition.

A Banach analytic space $X$ is said to have the Hartogs extension property (HEP) if every holomorphic map from a Riemann domain $\Omega$ over a Banach space $B$ with a Schauder basis into $X$ can be extended holomorphically to $\hat{\Omega}$, the envelope of holomorphy of $\Omega$.

We prove the following.

Theorem 2.1. Let $X$ be a Banach analytic space which is an increasing union of pseudoconvex domains. Assume that $X$ contains no complex lines. Then $X$ has the HEP.

Finally in this note we frequently make use of the definition and properties of the Kobayashi pseudodistance in Banach analytic spaces as in [18], [19].

\section{§1. ExTENDing HOLOMORPHiC MAPS THROUGH HYPERSURFACES IN COMPLEX BANACH MANIFOLDS}

First we prove the Kwack theorem [10] in infinite dimensions.

1.1 Theorem. Let $X$ be a hyperbolic Banach space and $f: Z \backslash H \rightarrow X$ a holomorphic map, where $Z$ is a Banach manifold and $H$ is a hypersurface in $Z$. Assume that for every $z \in H$ there exists a sequence $\left\{z_{n}\right\} \subset Z \backslash H$ converging to $z$ such that the sequence $\left\{f\left(z_{n}\right)\right\}$ converges to $x_{z} \in X$. Then $f$ extends holomorphically to $Z$. 
Proof. (i) First consider the case where $Z=\Delta$ and $H=\{0\}$. Choose a pseudoconvex coordinate neighbourhood $W$ of $x_{0}$ in $X$ which is isomorphic to an analytic subset of an open ball in a Banach space $B$. Let $V=W / 2$.

The problem is to show that, for a suitable positive number $\sigma$, the small punctured disc $\{z \in \Delta: 0<|z|<\sigma\}$ is mapped into $W$ by $f$. By taking a subsequence of $\left\{z_{n}\right\}$ if necessary, we may assume that the sequence $\left\{\left|z_{n}\right|\right\}$ is monotone decreasing. Consider the set of integers $n$ such that the image of the annulus $\left|z_{n+1}\right|<|z|<\left|z_{n}\right|$ by $f$ is not entirely contained in $V$. If this set of integers is finite, then $f$ maps a small punctured disc $0<|z|<\sigma$ into $\bar{V}$. Assuming that this set of integers is infinite, we shall obtain a contradiction. By taking a subsequence, we may assume also that, for every $n$, the image of the annulus $\left|z_{n+1}\right|<|z|<\left|z_{n}\right|$ by $f$ is not entirely contained in $V$.

For each $n$, put

$$
\begin{gathered}
r_{n}=\inf \left\{r<\left|z_{n}\right|: f\left(r<|z|<\left|z_{n}\right|\right) \subset V\right\}, \\
s_{n}=\sup \left\{r>\left|z_{n}\right|: f\left(\left|z_{n}\right|<|z|<r\right) \subset V\right\}, \\
\alpha_{n}=\left\{z \in \Delta:|z|=r_{n}\right\}, \\
\gamma_{n}=\left\{z \in \Delta:|z|=\left|z_{n}\right|\right\}, \\
\beta_{n}=\left\{z \in \Delta:|z|=s_{n}\right\} .
\end{gathered}
$$

Since $d_{\Delta^{*}}\left(\alpha_{n}\right)+d_{\Delta^{*}}\left(\gamma_{n}\right)+d_{\Delta^{*}}\left(\beta_{n}\right) \rightarrow 0$ and by the distance decreasing principle, it follows that

$$
d_{X} f\left(\alpha_{n}\right)+d_{X} f\left(\gamma_{n}\right)+d_{X} f\left(\beta_{n}\right) \rightarrow 0 \text { as } n \rightarrow \infty
$$

Put $K=\bigcup_{n=1}^{\infty} f\left(\gamma_{n} \cup \gamma_{n+1}\right)$.

By the maximum principle, we have

$$
\widehat{K}_{P S H(W)} \supset \bigcup_{n \geq 2} f\left(\alpha_{n} \cup \beta_{n}\right) .
$$

Hence $\bigcup_{n \geq 2} f\left(\alpha_{n} \cup \beta_{n}\right)$ is relatively compact in $\bar{V}$. By the relative compactness of $\bigcup_{n \geq 2} f\left(\alpha_{n} \cup \beta_{n}\right)$ and since $d_{X} f\left(\alpha_{n}\right) \rightarrow 0$ and $d_{X} f\left(\beta_{n}\right) \rightarrow 0$, without loss of generality we may assume that $\left\{f\left(\alpha_{n}\right)\right\} \rightarrow x_{1}$ and $\left\{f\left(\beta_{n}\right)\right\} \rightarrow x_{2}$. By the definition of $r_{n}$ and $s_{n}$ it follows that $x_{1}, x_{2} \in \partial V$ and hence $x_{1}, x_{2} \neq x_{0}$. Choose a continuous linear functional $u$ on $B$ such that $u\left(x_{1}\right), u\left(x_{2}\right) \neq u\left(x_{0}\right)=0$.

Since $f\left(\bar{\Delta}_{r_{n} s_{n}}\right) \subset \bar{V} \subset W$, there exist $\tilde{r}_{n}<r_{n}<s_{n}<\tilde{s}_{n}$ such that $f\left(\Delta_{\tilde{r}_{n} \tilde{s}_{n}}\right) \subset$ $W$, where $\bar{\Delta}_{r_{n} s_{n}}=\left\{z \in \mathbb{C}: r_{n} \leq|z| \leq s_{n}\right\}$ and $\Delta_{\tilde{r}_{n} \tilde{s}_{n}}=\left\{z \in \mathbb{C}: \tilde{r}_{n}<|z|<\tilde{s}_{n}\right\}$.

Consider the holomorphic function $\sigma_{n}=\left.u \circ f\right|_{\Delta_{\tilde{r}_{n} \tilde{s}_{n}}}$. Since $\left\{\sigma_{n}\left(\beta_{n}\right)\right\} \rightarrow u\left(x_{2}\right)$, we have $\forall \epsilon>0, \exists N, \forall n \geq N, \forall \theta:\left|\sigma_{n}\left(s_{n} e^{i \theta}\right)-u\left(x_{2}\right)\right|<\epsilon$. Applying the maximum principle to the function $z \mapsto \sigma_{n}(z)-u\left(x_{2}\right)$ on the annulus $\left\{z \in \mathbb{C}: \tilde{r}_{n}<|z| \leq s_{n}\right\}$, in particular the circle $\left\{z \in \mathbb{C}:|z|=\left|z_{n}\right|\right\} \subset \Delta_{\tilde{r}_{n} s_{n}}=\left\{z \in \mathbb{C}: \tilde{r}_{n}<|z|<s_{n}\right\}$, it implies that $\left|\sigma_{n}\left(\left|z_{n}\right| e^{i \theta}\right)-u\left(x_{2}\right)\right|<\epsilon$ for every $\theta$. Thus $u\left(x_{0}\right)=u\left(x_{2}\right)$. This is impossible. Hence $f$ extends holomorphically to $\Delta$.

(ii) Assume $H$ contains no singular points.

Without loss of generality we may assume that the manifold $Z$ has the form $U \times \Delta$, where $U$ is an open subset of a Banach space, and $H=U \times\{0\}$. 
For each $z \in U$ consider $f^{z}: \Delta^{*} \rightarrow X$ given by

$$
f^{z}(\lambda)=f(z, \lambda) \quad \text { for each } \lambda \in \Delta^{*} .
$$

Since $(z, 0) \in H$ there exists $\left\{\left(z_{n}, \lambda_{n}\right)\right\} \subset U \times \Delta^{*},\left\{\left(z_{n}, \lambda_{n}\right)\right\} \rightarrow(z, 0)$ such that $\left\{f\left(z_{n}, \lambda_{n}\right)\right\} \rightarrow x_{0}$. By applying the distance decreasing principle for the holomorphic map $f: U \times \Delta^{*} \rightarrow X$, we have

$$
d_{X}\left(f\left(z, \lambda_{n}\right), f\left(z_{n}, \lambda_{n}\right)\right) \leq d_{U \times \Delta^{*}}\left(\left(z_{n}, \lambda_{n}\right),\left(z, \lambda_{n}\right)\right)=d_{U}\left(z, z_{n}\right) \rightarrow 0 .
$$

It follows that $\left\{f\left(z, \lambda_{n}\right)\right\} \rightarrow x_{0}$.

By (i), $f^{z}$ extends to a holomorphic map $\hat{f}^{z}: \Delta \rightarrow X$. Define the map $\hat{f}$ : $U \times \Delta \rightarrow X$ by $\hat{f}(z, \lambda)=\hat{f}^{z}(\lambda)$ for $(z, \lambda) \in U \times \Delta$. Since $X$ is hyperbolic, $\hat{f}$ is continuous.

Indeed, let $(z, 0) \in H$ and $\left\{\left(z_{n}, \lambda_{n}\right)\right\} \subset U \times \Delta$ be such that $\left\{\left(z_{n}, \lambda_{n}\right)\right\} \rightarrow(z, 0)$. Choose $\left\{\tilde{\lambda}_{n}\right\} \subset \Delta^{*}$ such that $\left\{\tilde{\lambda}_{n}\right\} \rightarrow 0$. We have

$$
\begin{aligned}
& d_{X}\left(\hat{f}\left(z_{n}, \lambda_{n}\right), \hat{f}(z, 0)\right) \leq d_{X}\left(\hat{f}\left(z_{n}, \lambda_{n}\right), \hat{f}\left(z_{n}, \tilde{\lambda}_{n}\right)\right)+d_{X}\left(\hat{f}\left(z_{n}, \tilde{\lambda}_{n}\right), \hat{f}\left(z, \tilde{\lambda}_{n}\right)\right) \\
& +d_{X}\left(\hat{f}\left(z, \tilde{\lambda}_{n}\right), \hat{f}(z, 0)\right) \\
& =d_{X}\left(\hat{f}^{z_{n}}\left(\lambda_{n}\right), \hat{f}^{z_{n}}\left(\tilde{\lambda}_{n}\right)\right)+d_{X}\left(f\left(z_{n}, \tilde{\lambda}_{n}\right), f\left(z, \tilde{\lambda}_{n}\right)\right)+d_{X}\left(\hat{f}^{z}\left(\tilde{\lambda}_{n}\right), \hat{f}^{z}(0)\right) \\
& \leq d_{\Delta}\left(\lambda_{n}, \tilde{\lambda}_{n}\right)+d_{U}\left(z_{n}, z\right)+d_{\Delta}\left(\tilde{\lambda}_{n}, 0\right) \rightarrow 0 \quad \text { as } n \rightarrow \infty \text {. }
\end{aligned}
$$

Thus $\hat{f}$ is a holomorphic extension of $f$.

(iii) Let $\alpha \in H$ be an arbitrary point of $H$. By Ramis [14, p.14, Corollaire] there exists a neighbourhood $U$ which is isomorphic to a neighbourhood $V \times \Delta \mathbf{e}$ of $0 \in B$ and a Weierstrass polynomial

$$
P(x, \lambda)=\lambda^{p}+a_{p-1}(x) \lambda^{p-1}+\cdots+a_{0}(x)
$$

such that Zero $(P)=H \cap U$, for some decomposition $B=E \oplus C \mathbf{e}$ of $B$.

We have $U \cap H=\operatorname{Zero}(P)=\left(\operatorname{Zero}\left(\frac{\partial P}{\partial \lambda}\right) \cap \operatorname{Zero}(P)\right) \cup\left(\operatorname{Zero}(P) \backslash \operatorname{Zero}\left(\frac{\partial P}{\partial \lambda}\right)\right)$. By (ii) $f$ extends to the holomorphic map $f_{1}: U \backslash \operatorname{Zero}\left(\frac{\partial P}{\partial \lambda}\right) \rightarrow X$. We now prove, for every $z_{0} \in \operatorname{Zero}\left(\frac{\partial P}{\partial \lambda}\right)$, there exists $\left\{z_{n}\right\} \subset U \backslash \operatorname{Zero}\left(\frac{\partial P}{\partial \lambda}\right)$ converging to $z_{0}$ such that $\left\{f_{1}\left(z_{n}\right)\right\}$ converges to $x_{z_{0}} \in X$.

Indeed, by hypothesis there exists $\left\{z_{n}^{\prime}\right\} \subset U \backslash H$ converging to $z_{0}$ such that $\left\{f\left(z_{n}^{\prime}\right)\right\}$ converges to $x_{z_{0}} \in X$.

Choose $\left\{z_{n}\right\} \subset U \backslash\left(\operatorname{Zero}\left(\frac{\partial P}{\partial \lambda}\right) \cup H\right)$ such that $d_{U \backslash H}\left(z_{n}, z_{n}^{\prime}\right) \rightarrow 0$. Then the sequence $\left\{z_{n}\right\}$ satisfies the above requirement.

Repeating this process $f$ can be extended holomorphically to $U \backslash \operatorname{Zero}\left(\frac{\partial^{2} P}{\partial^{2} \lambda}\right), \ldots$, $U \backslash \operatorname{Zero}\left(\frac{\partial^{p} P}{\partial^{p} \lambda}\right)=U$. Q.E.D.

1.2 Theorem. Let $X$ be a hyperbolic Banach analytic space which is complete in the Cauchy mean, and $f: Z \backslash H \rightarrow X$ a holomorphic map, where $H$ is a hypersurface in a Banach analytic manifold $Z$. Assume that for every branch $H_{\alpha}$ of $H$ there exist $z_{\alpha} \in \operatorname{Reg}\left(H_{\alpha}\right)$ and a sequence $\left\{z_{n}^{\alpha}\right\}_{n=1}^{\infty} \subset Z \backslash H$ converging to $z_{\alpha}$ such that the sequence $\left\{f\left(z_{n}^{\alpha}\right)\right\}$ converges to $p_{\alpha} \in X$. Then $f$ extends holomorphically to $Z$.

Proof. (i) First of all we prove that $X$ satisfies the weakly disk-convex condition, i.e. if every sequence $\left\{\varphi_{n}\right\} \subset \operatorname{Hol}(\Delta, X)$ converges in $\operatorname{Hol}(\Delta, X)$ whenever the sequence $\left\{\left.\varphi_{n}\right|_{\Delta^{*}}\right\}$ converges in $\operatorname{Hol}\left(\Delta^{*}, X\right)$, where $\operatorname{Hol}(X, Y)$ denotes the space of all holomorphic maps from $X$ into $Y$ with the open-compact topology. 
Indeed, let $\left\{\varphi_{n}\right\} \subset \operatorname{Hol}(\Delta, X)$ be such that $\varphi_{n} \rightarrow \varphi$ in $\operatorname{Hol}\left(\Delta^{*}, X\right)$. Since

$$
\begin{aligned}
d_{X}\left(\varphi_{n}(0), \varphi_{m}(0)\right) & \leq d_{X}\left(\varphi_{n}(0), \varphi_{n}(z)\right)+d_{X}\left(\varphi_{n}(z), \varphi_{m}(z)\right)+d_{X}\left(\varphi_{m}(z), \varphi_{m}(0)\right) \\
& \leq 2 d_{\Delta}(0, z)+d_{X}\left(\varphi_{n}(z), \varphi_{m}(z)\right)
\end{aligned}
$$

and $\varphi_{n} \rightarrow \varphi$ in $\operatorname{Hol}\left(\Delta^{*}, X\right)$, it follows that $\left\{\varphi_{n}(0)\right\}$ converges to $x_{0} \in X$ as $n \rightarrow \infty$. Define the map $\widetilde{\varphi}: \Delta \rightarrow X$ by $\left.\widetilde{\varphi}\right|_{\Delta^{*}}=\varphi$ and $\widetilde{\varphi}(0)=x_{0}$. Since $X$ is hyperbolic, $\widetilde{\varphi}$ is continuous. This yields $\widetilde{\varphi} \in \operatorname{Hol}(\Delta, X)$ and hence $\varphi_{n} \rightarrow \widetilde{\varphi}$ in $\operatorname{Hol}(\Delta, X)$.

(ii) By (i) $X$ has the HEP (see [16] or [17]). Thus, it suffices to prove that $f$ extends holomorphically over $(Z \backslash H) \cup W$, where $W$ is an open subset of $Z$ such that $W \cap H_{\alpha}$ is not empty for every branch $H_{\alpha}$ of $H$.

(iii) Assume that $H_{\alpha}$ is a branch of $H$ and $z_{\alpha} \in \operatorname{Reg}\left(H_{\alpha}\right)$ as in the assumption of Theorem 1.2. We prove that $f$ extends holomorphically over an open neighbourhood $W_{\alpha}$ of $z_{\alpha}$ in $Z$.

Since the problem is local, we may assume that $Z=B \times \Delta$ with $H_{\alpha}=B \times\{0\}$, where $B$ is the open unit ball in a Banach space $E$, and let $z_{n}^{\alpha} \rightarrow z_{\alpha}=(x, 0)$. Let $F$ be an arbitrary finite dimensional subspace of $E$ containing $x$. We write $z_{n}^{\alpha}=\left(x_{n}^{\alpha}, \lambda_{n}^{\alpha}\right) \in B \times \Delta^{*}$. Let $P: E \rightarrow F$ be a continuous linear projection of $E$ onto $F$. We may assume that $P x_{n}^{\alpha} \in B \cap F$ for every $n \geq 1$.

Since $B$ is hyperbolic and $P x_{n}^{\alpha} \rightarrow P x=x$, we have

$$
\begin{aligned}
d_{X}\left(f\left(x_{n}^{\alpha}, \lambda_{n}^{\alpha}\right), f\left(P x_{n}^{\alpha}, \lambda_{n}^{\alpha}\right)\right) & =d_{X}\left(f_{\lambda_{n}^{\alpha}}\left(x_{n}^{\alpha}\right), f_{\lambda_{n}^{\alpha}}\left(P x_{n}^{\alpha}\right)\right) \\
& \leq d_{B}\left(x_{n}^{\alpha}, P x_{n}^{\alpha}\right) \rightarrow 0 \text { as } n \rightarrow \infty,
\end{aligned}
$$

where $f_{\lambda_{n}^{\alpha}}(x)=f\left(x, \lambda_{n}^{\alpha}\right)$. Hence $f\left(P x_{n}^{\alpha}, \lambda_{n}^{\alpha}\right) \rightarrow p_{\alpha}$ as $n \rightarrow \infty$. Choose a pseudoconvex coordinate neighbourhood $W$ of $p_{\alpha}$ in $X$ which is isomorphic to an open ball in a Banach space $B$. Let $V=W / 2$.

Consider $f_{F}=\left.f\right|_{(B \cap F) \times \Delta^{*}}$. Put $B_{F}=B \cap F$. By taking a subsequence of $\left\{z_{n}\right\}$ if necessary, we may assume that the sequence $\left\{\left|\lambda_{n}^{\alpha}\right|\right\}$ is monotone decreasing. By making use of the same argument in the proof of Theorem 1.1 (part (i)) suppose that, for every $n$, the image of the set $\frac{\partial B_{F}}{n} \times\left\{\left|\lambda_{n+1}^{\alpha}\right|<|z|<\left|\lambda_{n}^{\alpha}\right|\right\}$ by $f_{F}$ is not entirely contained in $V$. Put

$$
\begin{aligned}
& r_{n}=\inf \left\{r \in\left(0,\left|\lambda_{n}^{\alpha}\right|\right): f_{F}\left(\frac{\partial B_{F}}{n} \times \lambda\right) \subset V, \quad \forall \lambda \in \mathbb{C}, \text { such that } r<|\lambda|<\left|\lambda_{n}^{\alpha}\right|\right\}, \\
& s_{n}=\sup \left\{r \in\left(\left|\lambda_{n}^{\alpha}\right|, 1\right): f_{F}\left(\frac{\partial B_{F}}{n} \times \lambda\right) \subset V, \quad \forall \lambda \in \mathbb{C}, \text { such that }\left|\lambda_{n}^{\alpha}\right|<|\lambda|<r\right\} .
\end{aligned}
$$

Let $\alpha_{n}=\left\{\lambda \in \Delta:|\lambda|=r_{n}\right\}, \gamma_{n}=\left\{\lambda \in \Delta:|\lambda|=\left|\lambda_{n}^{\alpha}\right|\right\}, \beta_{n}=\left\{\lambda \in \Delta:|\lambda|=s_{n}\right\}$.

Then

$$
d_{X} f\left(\frac{\partial B_{F}}{n} \times \alpha_{n}\right)+d_{X} f\left(\frac{\partial B_{F}}{n} \times \gamma_{n}\right)+d_{X} f\left(\frac{\partial B_{F}}{n} \times \beta_{n}\right) \rightarrow 0
$$

as $n \rightarrow \infty$.

Consider the compact subset $K$ in $W$ given by

$$
K=\mathrm{Cl}\left(\bigcup_{n \geq 1} f\left(\left(\frac{\partial B_{F}}{n} \times \gamma_{n}\right) \cup\left(\frac{\partial B_{F}}{n} \times \gamma_{n+1}\right)\right)\right) .
$$


By the maximum principle we have

$$
\widehat{K}_{P S H(W)} \supset \bigcup_{n \geq 2} f\left(\left(\frac{\partial B_{F}}{n} \times \alpha_{n}\right) \cup\left(\frac{\partial B_{F}}{n} \times \beta_{n}\right)\right),
$$

and hence $\bigcup_{n \geq 2} f\left(\left(\frac{\partial B_{F}}{n} \times \alpha_{n}\right) \cup\left(\frac{\partial B_{F}}{n} \times \beta_{n}\right)\right)$ is relatively compact in $V$.

Without loss of generality we may assume that

$$
f\left(\frac{\partial B_{F}}{n} \times \alpha_{n}\right) \rightarrow \omega_{1}
$$

and

$$
f\left(\frac{\partial B_{F}}{n} \times \beta_{n}\right) \rightarrow \omega_{2} .
$$

By the definition of $r_{n}$ and $s_{n}$, it follows that $\omega_{1}, \omega_{2} \in \partial V$ and hence $\omega_{1}, \omega_{2} \neq p_{\alpha}$. Choose a continuous linear functional $u$ such that $u\left(\omega_{1}\right), u\left(\omega_{2}\right) \neq u\left(p_{\alpha}\right)=0$.

Since $f\left(\frac{\partial B_{F}}{n} \times \bar{\Delta}_{r_{n} s_{n}}\right) \subset \bar{V} \subset W$, there exist $\tilde{r}_{n}<r_{n}<s_{n}<\tilde{s}_{n}$ such that $f\left(\frac{\partial B_{F}}{n} \times \Delta_{\tilde{r}_{n} \tilde{s}_{n}}\right) \subset W$.

Take a point $x_{0} \in \partial B_{F}$. Consider the holomorphic function

$$
\sigma_{n}(\lambda)=u \circ f\left(\frac{x_{0}}{n}, \lambda\right) \text { on } \Delta_{\tilde{r}_{n} \tilde{s}_{n}} .
$$

It is easy to see that $\left\{\sigma_{n}\left(\gamma_{n}\right)\right\} \rightarrow u\left(p_{\alpha}\right)=0$ and $\left\{\sigma_{n}\left(\beta_{n}\right)\right\} \rightarrow u\left(\omega_{2}\right)$.

By making use of the same argument as in Theorem 1.1, we get a contradiction. Thus $f_{F}$ is extended holomorphically to $B_{F} \times \Delta$.

By (iii) the family $\left\{f_{F}\right\}$ defines a Gateaux holomorphic map $\hat{f}: Z \backslash S(H) \rightarrow X$, where $S(H)$ denotes the singular locus of $H$.

Since $[3]$

$$
d_{B \times \Delta}(u, v)=\inf \left\{d_{B \cap F \times \Delta}(u, v): F \ni u, v \text { and } \operatorname{dim} F<\infty\right\},
$$

we have

$d_{X}(\hat{f}(u), \hat{f}(v)) \leq \inf \left\{d_{B \cap F \times \Delta}(u, v): F \ni u, v\right.$ and $\left.\operatorname{dim} F<\infty\right\}=d_{B \times \Delta}(u, v)$.

Hence $\hat{f}$ is continuous. This yields the holomorphicity of $\hat{f}$ Q.E.D.

1.3. Remark. Theorem 1.2 was proved by Fujimoto [5] when $Z$ is a finite-dimensional complex space and $X$ is a taut complex space. Howeover, since tautness is not defined in the infinite-dimensional case, the assumption on the complete hyperbolicity of $X$ is a natural substitute.

\section{$\S 2$. HARTOGS HOLOMORPHIC EXTENSION}

We now prove the following

2.1. Theorem. Let $X$ be a Banach analytic space which is an increasing union of pseudoconvex domains. Assume that $X$ contains no complex lines. Then $X$ has the HEP. 
Proof. (i) First we assume that $X$ is pseudoconvex. Let $f: \Omega \longrightarrow X$ be a holomorphic map. Consider the commutative diagram

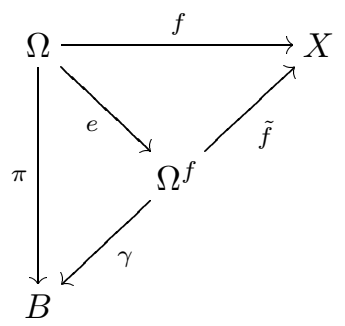

where $\Omega^{f}$ is the domain of existence of $f$ with the canonical extension $\tilde{f}: \Omega^{f} \rightarrow X$ and $e, \gamma, \pi$ are locally biholomorphic canonical maps.

We need the following.

2.2. Lemma. The map $\tilde{f}: \Omega^{f} \rightarrow X$ is locally pseudoconvex, i.e. for every $x \in$ $X$ there exists a pseudoconvex neighbourhood $U$ of $x$ in $X$ such that $\tilde{f}^{-1}(U)$ is pseudoconvex.

Proof. Given $x \in X$. Choose a neighbourhood $V$ of $x$ in $X$ which is isomorphic to an analytic set in an open ball of a Banach space.

Consider the restriction $\left.\tilde{f}\right|_{\tilde{f}^{-1}(V)}$. Let $g:{ }^{\wedge} \tilde{f}^{-1}(V) \rightarrow V$ be a holomorphic extension of $\left.\tilde{f}\right|_{\tilde{f}^{-} 1(V)}$ to the envelope of holomorphy ${ }^{\wedge} \tilde{f}^{-1}(V)$ of $\tilde{f}^{-1}(V)$. Since $\Omega^{f}$ is the domain of existence of $f$, it follows that ${ }^{\wedge} \tilde{f}^{-1}(V) \subset \Omega^{f}$.

On the other hand, from the relation

$$
\tilde{f}\left(\wedge \tilde{f}^{-1}(V)\right)=g\left(\wedge \tilde{f}^{-1}(V)\right) \subset V
$$

we have $\tilde{f}^{-1}(V)=\wedge \tilde{f}^{-1}(V)$.

Consequently $\tilde{f}: \Omega^{f} \rightarrow X$ is locally pseudoconvex.

In order to show that $\Omega^{f}={ }^{\wedge} \Omega$ it remains to check that $\Omega^{f}$ satisfies the weakly disc-convex condition.

Indeed, let $\left\{\sigma_{k}\right\} \subset \operatorname{Hol}\left(\Delta, \Omega^{f}\right)$ be such that the sequence $\left\{\left.\sigma_{k}\right|_{\Delta^{*}}\right\}$ converges to $\sigma$ in $\operatorname{Hol}\left(\Delta^{*}, \Omega^{f}\right)$. Since $X$ is pseudoconvex and $X$ contains no complex lines, $X$ satisfies the weakly disc-convex condition (see [17, Proposition 2.3]). Thus the sequence $\left\{\tilde{f} \circ \sigma_{k}\right\} \subset \operatorname{Hol}(\Delta, X)$ converges to $\tilde{f} \circ \sigma$ in $\operatorname{Hol}(\Delta, X)$. Choose a pseudoconvex neighbourhood $V$ of $\overline{\tilde{f} \circ \sigma}(0)$ which is isomorphic to an analytic set in an open ball of a Banach space and $\tilde{f}^{-1}(V)$ is pseudoconvex. Since $\tilde{f}^{-1}(V)$ is a pseudoconvex Riemann domain over a Banach space $B$ with a Schauder basis, $\tilde{f}^{-1}(V)$ is a domain of holomorphy. It is easy to see that there exist $k_{0}$ and $\varepsilon>0$ such that $\left(\tilde{f} \circ \sigma_{k}\right)\left(\Delta_{\varepsilon}\right) \subset V$ for every $k>k_{0}$ and $\overline{\tilde{f} \circ \sigma}\left(\Delta_{\varepsilon}\right) \subset V$, where $\Delta_{\varepsilon}=\{z \in \mathbb{C}:|z|<\varepsilon\}$. Hence $\sigma_{k}\left(\Delta_{\varepsilon}\right) \subset \tilde{f}^{-1}(V)$ for every $k>k_{0}$. It follows that $\left\{\left.\sigma_{k}\right|_{\Delta_{\varepsilon}}\right\} \rightarrow \bar{\sigma}$ in $\operatorname{Hol}\left(\Delta_{\varepsilon}, \tilde{f}^{-1}(V)\right)$ (see [6, Theorem 5 and Lemma 6]. Thus the sequence $\left\{\sigma_{k}\right\}$ is convergent in $\operatorname{Hol}\left(\Delta, \Omega^{f}\right)$.

(ii) Assume that $X=\bigcup_{n=1}^{\infty} X_{n}$ where $X_{n}$ are pseudoconvex domains and $X_{1} \subset$ $X_{2} \subset \ldots$

Put $\Omega_{n}=f^{-1}\left(X_{n}\right)$ for each $n \geq 1$. 
By (i), for each $n \geq 1$, the map $f_{n}=\left.f\right|_{\Omega_{n}}$ extends to a holomorphic map $\hat{f}_{n}:^{\wedge} \Omega_{n} \rightarrow X_{n}$.

It is easy to see that for each $n \geq 1$ there exists a unique locally biholomorphic map $e_{n}:^{\wedge} \Omega_{n} \rightarrow^{\wedge} \Omega_{n+1}$ such that the following diagram is commutative:

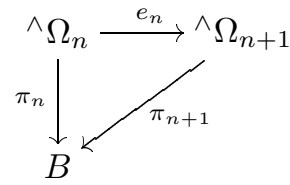

and $\hat{f}_{n+1} e_{n}=\hat{f}_{n}$ for $n \geq 1$, where $\pi_{n}:^{\wedge} \Omega_{n} \rightarrow B$ defines ${ }^{\wedge} \Omega_{n}$ as a Riemann domain over $B$. Thus we can define maps $\tilde{f}: \widetilde{\Omega}=\lim _{\operatorname{ind}}{ }^{\wedge} \Omega_{n} \rightarrow X$ and $\pi: \widetilde{\Omega} \rightarrow B$ by $\left.\tilde{f}\right|_{\wedge \Omega_{n}}=\hat{f}_{n}$ for all $n \geq 1$ and $\left.\pi\right|_{\wedge} \Omega_{n}=\pi_{n}$ for all $n \geq 1$. Since $\pi_{n}$ is a local homeomorphism for $n \geq 1$, it follows that $\pi$ is also a local homeomorphism.

Moreover, we have $d_{n}(z) \leq d_{n+1}\left(e_{n}(z)\right)$ for all $z \in \wedge^{\wedge} \Omega_{n}$ and $n \geq 1$, where $d_{n}$ denotes the boundary distance with respect to $\pi_{n}:{ }^{\wedge} \Omega_{n} \rightarrow B$ for each $n \geq 1$.

Since ${ }^{\wedge} \Omega_{n}$ is pseudoconvex, $-\log d_{n}$ is plurisubharmonic for all $n \geq 1$. Hence the function $-\log d(z)=\lim _{n \rightarrow \infty}-\log d_{n}(z)$, for every $z \in \widetilde{\Omega}$, is plurisubharmonic. This means that $\widetilde{\Omega}$ is pseudoconvex and hence $\widetilde{\Omega}$ is a domain of holomorphy.

This yields $\widetilde{\Omega}={ }^{\wedge} \Omega$. The theorem is proved. $\quad$ Q.E.D.

2.3. Remark. There exists a complex manifold $X$ which is not pseudoconvex such that $X=\bigcup_{n=1}^{\infty} X_{n}$, where $X_{n}$ is Stein.

Indeed, as in [4] for each $n$ we put

$$
X_{n}=\left\{(z, \omega, \eta) \in \mathbb{C}^{3}: \omega \eta=p_{n}(z), p_{n}(z)=\prod_{k=1}^{n}\left(z-\frac{1}{k}\right)\right\} .
$$

Obviously, $X_{n}$ are closed submanifolds of $\mathbb{C}^{3}$ and hence, $X_{n}$ are Stein. For each $n$, consider the map $\gamma_{n}: X_{n} \rightarrow X_{n+1}$ defined as follows:

$$
\gamma_{n}(z, \omega, \eta)=\left(z, \omega, \eta \cdot\left(z-\frac{1}{n+1}\right)\right) .
$$

Clearly, $\gamma_{n}$ is biholomorphic from $X_{n}$ onto $X_{n+1} \backslash\left\{\frac{1}{n+1} \times \mathbb{C}^{2}\right\}$. Thus we can define $X=\lim _{\rightarrow}\left(X_{n}, \gamma_{n}\right)$. We shall prove that $X$ is not pseudoconvex. For the converse case, we assume that $X$ is pseudoconvex and hence, in our case $X$ satisfies the weakly disc-convex condition. Let $\left\{f_{n}\right\} \subset \operatorname{Hol}(\Delta, X)$ be a sequense of maps defined by

$$
f_{n}(\lambda)=\left(\lambda, \lambda-\frac{1}{n+1}, p_{n}(\lambda)\right) .
$$

Then $f_{n}(\Delta) \subset X_{n+1}$. We prove that $\left\{f_{n}\right\}$ is uniformly convergent in $\operatorname{Hol}\left(\Delta^{*}, X\right)$. For each $k$, consider $f_{n}^{k} \in \operatorname{Hol}\left(\Delta_{\frac{1}{k+1}, 1}, X\right)$ defined by

$$
f_{n}^{k}(\lambda)=\left(\lambda, \lambda-\frac{1}{n+1}, \frac{p_{k}(\lambda)}{\lambda-\frac{1}{n+1}}\right), \quad \lambda \in \Delta_{\frac{1}{k+1}, 1},
$$

where

$$
\Delta_{\frac{1}{k+1}, 1}=\left\{z \in \Delta: \frac{1}{k+1}<|z|<1\right\} .
$$


Note that

$$
f_{n}^{k} \in \operatorname{Hol}\left(\Delta_{\frac{1}{k+1}, 1}, X_{k}\right)
$$

For every $n>k,\left\{f_{n}^{k}\right\}$ converges in $\operatorname{Hol}\left(\Delta_{\frac{1}{k+1}}, 1, X\right)$ to the map $f^{k}$ given by $f^{k}(\lambda)=\left(\lambda, \lambda, \frac{p_{k}(\lambda)}{\lambda}\right)$. On the other hand, since

$$
\gamma_{n \circ} \gamma_{n-1} \ldots \circ \gamma_{k_{\circ}} f_{n}^{k}=f_{n} \text { for every } n, k \geq 1,
$$

we have $\gamma_{q} \ldots \gamma_{p} f^{p}=f^{q}$, where $p, q$ are natural numbers with $p<q$. Thus we can define a map $f: \Delta^{*} \rightarrow X$ by setting $f(z)=f^{k}(z)$ for every $z \in \Delta_{\frac{1}{k+1}, 1}$. Since $\frac{1}{k+1} \rightarrow 0$, the sequence $\left\{f_{n}\right\}$ converges to $f$ in $\operatorname{Hol}\left(\Delta^{*}, X\right)$. By hypothesis, $\left\{f_{n}\right\}$ converges to $f$ in $\operatorname{Hol}(\Delta, X)$. Consider $\bar{\Delta}_{\varepsilon}=\{z \in \mathbb{C}:|z| \leq \varepsilon\}, \varepsilon \in(0,1)$. Since $\left\{f_{n}\right\}$ is uniformly convergent on $\bar{\Delta}_{\varepsilon}$, it follows that $\bigcup_{n=1}^{\infty} f_{n}\left(\bar{\Delta}_{\varepsilon}\right)$ is compact. Since $X=\bigcup_{n=1}^{\infty} X_{k}, X_{k} \subset X_{k+1}$ and $X_{k}$ is open in $X$ for every $k \geq 1$, there exists $k_{0}$ such that $\bigcup_{n=1}^{\infty} f_{n}\left(\overline{\Delta_{\varepsilon}}\right) \subset X_{k_{0}}$. Hence

$$
f^{k_{0}}(\lambda)=\left(\lambda, \lambda, \frac{p_{k_{0}}(\lambda)}{\lambda}\right) \text { for all } \lambda \in \Delta_{\varepsilon}^{*} .
$$

Thus $f^{\left(k_{0}\right)}(\lambda)$ can be extended holomorphically to $\Delta_{\varepsilon}$. This is impossible, because $p_{k_{0}}(0) \neq 0$. Hence $X$ is not pseudoconvex.

\section{REFERENCES}

1. T. Barth, The Kobayashi distance induces the standard topology, Proc. Amer. Math. Soc. 35 (1972), No 2, 439-441. MR 46:5668

2. R. Brody, Compact manifolds and hyperbolicity, Trans. Amer. Math. Soc. 235 (1978), 213-219. MR 57:10010

3. S. Dineen, R. Timoney and J. P. Vigué, Pseudodistances invariantes sur les domaines d'un espace localement convexe, Ann. Nor. Sup. Pisa 12 (1985), 515-529. MR 88b:32054

4. J. E. Fornaess, An increasing sequence of Stein manifolds whose limit is not Stein, Math. Ann. 223 (1976), 275-277. MR 54:5498

5. H. Fujimoto, On holomorphic maps into a taut complex space, Nagoya Math. J. 46 (1972), 49-61. MR 46:9375

6. Y. Hervier, On the Weierstrass problem in Banach spaces, Proc. on Infinite Dimensional Holomorphy, Lecture Notes in Math. 364 (1974), 157-167. MR 53:1266

7. S. M. Ivashkovicz, The Hartogs phenomenon for holomorphically convex Kähler manifolds, English transl. : Math. USSR Izvestiya 29 (1987), 225-232.

8. P. Kiernan, Extensions of holomorphic maps, Trans. Amer. Math. Soc. 172 (1972), 347-355. MR 47:7066

9. S. Kobayashi, Hyperbolic Manifolds and Holomorphic Mappings, Marcel Dekker, New York 1970. MR 43:3503

10. M. Kwack, Generalizations of the big Picard theorem, Ann. Math. 90 (1969), No 2, 9-22. MR 39:4445

11. S. Lang, Introduction to Complex Hyperbolic Spaces, Springer - Verlag, 1987. MR 88f:32065

12. P. Mazet, Analytic Sets in Locally Convex Spaces, Math. Studies, North - Holland, v. 121, 1987. MR 86i: 32012

13. J. Mujica, Complex Analysis in Banach Spaces, Math. Studies, North - Holland, v. 120, 1986. MR 88d:46084

14. J.P. Ramis, Sous-ensembles Analytiques d'une Variete Banachique Complexe, SpringerVerlag, 1970. MR 45:2205 
15. B. Shiffman, Extension of holomorphic maps into Hermitian manifolds, Math. Ann. 194 (1971), 249-258. MR 45:598

16. B. D. Tac, Extending holomorphic maps in infinite dimension, Ann. Polon. Math. 54 (1991), 241-253.

17. Do Duc Thai and Nguyen Le Huong, On the disc - convexity of Banach analytic manifolds, Ann. Polon. Math. 69 (1998), 1-11. CMP 98:14

18. E. Vesentini and T. Franzoni, Holomorphic Maps and Invariant Distances, North - Holland, Math. Studies 40, Amsterdam 1980. MR 82a:32032

19. E. Vesentini, Invariant distances and invariant differential metric in locally convex spaces, Spectral theory Banach centre Publication U.8 P.W.N., Polish Sci. Publisher. Warsaw 1982, 493-512. MR 85d:32049

Department of Mathematics, Vietnam National University, Institute of Pedagogy, Cau Giay - Tu Liem, Hanoi, Vietnam

E-mail address: ddthai@netnam.org.vn 